\title{
Competencias investigativas de estudiantes de $V$ año de Economía en modalidad de graduación, FAREM Estelí
}

\section{Research competences in the $\mathrm{V}$ year students of Economy, FAREM Estelí}

Samaria Ilù Alonso Valenzuela ${ }^{1}$

\section{RESUMEN}

En el presente ensayo se aborda las competencias que presentan estudiantes de la carrera de economía de la FAREM Estelí, en la realización del trabajo final de investigación. Las competencias son habilidades y destrezas básicas e genéricas que un estudiante debe de tener en investigación. Las competencias básicas se refieren al entendimiento sobre los fenómenos sociales y económicos; y las genéricas se refiere a las competencias que el estudiante debe de tener para poder aplicar los conocimientos en el ámbito de la investigación; tales como capacidad de análisis, organización, planificación, gestión de la información, gestión del trabajo en equipo, comportamiento ético, uso de las tecnologías de la información, entre otros. Las competencias que se adquieren al largo de la carrera deben ser aplicadas a cada una de las fases de la investigación; desde la fase inicial con el planteamiento del tema de investigación, hasta la presentación de su trabajo final.

Palabras clave: competencias; investigación; estudiantes; economía.

\begin{abstract}
This essay focus on the competences presented by the students of Economy bachelor at FAREM Estelí during the final research paper. The competences are basic abilities and skills that a students should have in a research. The basic and generic skills are related to the understanding of the social and economic phenomenon and the generic ones are the competences that the students should have in order to use all their research knowledge such as analysis skills, organization, planning, information usage, team work, ethic, IT skills, among others. All the skills that students acquire during the bachelor must be applied in each of the research phases, from the beginning with the research question until the final presentation.
\end{abstract}

Keywords: competences; research; students; economy.

DOI: https://doi.org/10.5377/multiensayos.v3i6.9684

Recibido: 01 de diciembre de 2017

Aceptado: 15 de diciembre de 2017

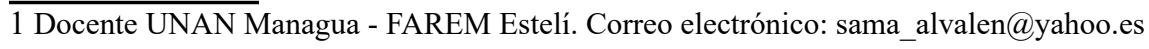




\section{INTRODUCCIÓN}

El Seminario de Graduación consiste en el análisis de temas propios del área del conocimiento, con el fin de dar propuestas de solución a las demandas de determinado sector socioeconómico. Tiene como propósito la adquisición de nuevos conocimientos, el desarrollo de habilidades y la formación de valores y actitudes necesarias para la preparación del profesional que demanda la sociedad nicaragüense. Se realiza bajo la conducción de un docente universitario y demanda trabajo independiente por parte de los estudiantes.

Las temáticas a investigar en seminario de graduación se derivan de las líneas de investigación de la UNAN MANAGUA, para la carrera de economía las líneas de investigación son tres, los cuales responden a las áreas disciplinares de la carrera de economía, tales como Macroeconomía, Microeconomía e instrumental de análisis de modelos económicos.

En el perfil de la carrera de economía se fundamenta que el profesional de la economía como investigador debe desarrollar con rigurosidad científica el análisis e interpretación de los fenómenos estudiados siendo consecuente con su visión epistemológica. (Universidad Nacional Autònoma de Nicaragua, 2012).

En la carrera de economía se imparten diferentes asignaturas que dan salida al eje de investigación planteado en el perfil de la carrera y que permite fortalecer las habilidades y destrezas de los estudiantes en el eje de investigación, tales como: técnicas de investigación documental, metodología de la investigación, investigación aplicada y modalidad de graduación. Así mismo, se imparten asignaturas que culminan con trabajo de curso.

El objetivo del presente ensayo es identificar las competencias que deben presentar los estudiantes de $\mathrm{V}$ año de Economía en la realización del trabajo de investigación como una forma de culminación de estudios.

\section{DESARROLLO}

Si bien es cierto, que las asignaturas de plan de estudio de la carrera de economía están planteadas por el enfoque por objetivos, el estudiante debe poder ser capaz de realizar diferentes desarrollar cuatro áreas: saber, saber hacer, saber ser y saber transferir y a la vez vincular estos saberes, lo cuales están planteados en el enfoque por competencia. (Càzares Aponte \& Cueves de la Garza, 2008).

Existen un sinnúmero de definiciones de competencias, podemos mencionar algunas: Tremblay (citado en Zabala \& Arnau, 2008), entiende la competencia como un sistema de conocimientos, conceptuales y de procedimiento, organizados en esquemas operacionales y que permiten, dentro 
de un grupo de situaciones la identificación de tareas-problemas y su resolución por una acción eficaz.

Competencia es sinónimo de capacidad, atributo, habilidad o destreza. Todos estos conceptos se relacionan con la persona y con lo que ésta es capaz de lograr. Pero tienen también significados más específicos. La palabra competencia se compone de tres elementos: Conocimientos, habilidades y rasgo de la personalidad. (Tovar, 2011).

Según Zabala \& Arnau (2008) tomando en cuenta las definiciones de competencias las ideas principales se pueden resumir en la siguiente tabla:

Tabla 1. ¿Qué es la Competencia?

Es la capacidades o habilidad Qué La existencia en las estructuras cognitivas de la persona de las condiciones y recursos para actuar, la capacidad, la habilidad, el dominio, la aptitud.

\section{De efectuar tareas o hacer frente a} situaciones diversas

Para que

Asumir un rol determinado; una ocupación, respecto a los niveles requeridos; una tarea específica; realizar acciones; participar en la vida política, social y cultural de la sociedad; cumplir con las exigencias complejas; resolver los problemas de la vida real; hacer frente a un tipo de situación.

De forma eficaz

De qué manera

Capacidad efectiva, de forma exitosa; ejercicio eficaz; movilizando a conciencia y de manera a la vez rápida, pertinente y creativa

En un contexto determinado

Dónde

Una actividad plenamente identificada; en un contex to determinado; en una situación determinada, en un ámbito o escenario de la actividad humana

Y para ello es necesario movilizar actitudes, habilidades y conocimientos

Varios recursos cognitivos; conocimientos, destrezas y características individuales; cualidades capacidades, aptitudes, conocimientos teóricos y metodológicos; comportamientos, facultad de análisis, toma de decisiones, transmisión de información, habilidades prácticas, valores éticos, entre otros.

Los estudiantes a la hora de realizar su trabajo de investigación deben se contar con ciertas competencias, tales como:

1. Competencias básicas: son aquellas relacionadas a conocimientos fundamentales del desenvolvimiento de la sociedad (Irias, 2012), esto es importante en los estudiantes de economía, ya que la economía tiene como objetivo principal entender las dinámicas sociales y su actuar en el ámbito económico. Para poder realizar investigación económica, los estudiantes 
deben entender la dinámica social en los procesos de producción, intercambio, distribución y consumo; y como esta dinámica afecta el sistema económico en su totalidad. Este aspecto es fundamental para poder definir y delimitar el tema de investigación, y en este aspecto los estudiantes presentan una dificultad para poder articular los temas de investigación a la dinámica económica y social.

2. Competencias genéricas: se relaciona con comportamientos y actitudes de labores propias de diferentes ámbitos de producción. Se caracterizan de aumentar la posibilidad de empleabilidad; esto se relaciona con las diferentes competencias que se adquieren adaptase a los entornos. Se pueden clasificar en (Irias, 2012):

- Competencias instrumentales, de orden metodológico o de procedimiento, tales como la capacidad de análisis y síntesis, de organización y planificación, y de gestión de información.

Para la realización de un trabajo de investigación se requiere efectuar un proceso de investigación exhaustivo y serio respecto al objeto de estudio seleccionado. Realizar un trabajo de investigación exige al estudiante aumentar su nivel de conocimiento, trabajar con más independencia y tener mayor sentido de dirección personal. (Blaxter, Cristina, \& Malcom, 2008). Para poder realizar el trabajo de investigación el estudiante debe ser capaz de poder desarrollar habilidades para planificar cada fase de la investigación.

- Competencias personales: los cuales incluyen ciertas habilidades para trabajar en equipo interdisciplinario y multidisciplinario, habilidades en las relaciones interpersonales, reconocimiento a la diversidad y multiculturalidad, razonamiento crítico y comportamiento ético.

Los estudiantes deben tener capacidad de coordinarse y organizarse con el grupo de investigación, poder entender las realidades sociales a través de un razonamiento critico de comportamiento de los fenómenos económico-sociales.

- Competencias sistémicas: Incluye la capacidad del aprendizaje autónomo, adaptación a nuevas situaciones, creatividad, liderazgo, conocimiento de otras culturas y costumbres, iniciativa y espíritu emprendedor, motivación por la calidad y sensibilidad para temas medioambientales.

- Otras competencias, tales como capacidad de aplicar los conocimientos a la práctica, uso de internet como medio de comunicación y como fuente de información, experiencias previas, capacidad

Para llevar a cabo la investigación deben tomar en cuenta ciertos aspectos para los cuales deben de poseer habilidades y destrezas: 
- Elegir un tema de investigación: Elegir un tema es la decisión más importante de todas las que se deben tomar a la hora de investigar. Aquí los estudiantes deben ser capaces de analizar los fenómenos sociales y económicos, así mismo debe ser capaz de poder vincular el campo económico al objeto de estudio. Uno de los problemas que con más frecuencia se ven enfrentados los estudiantes de economía es poder extraer un tema económico y aplicarlo a la realidad económica local.

- Trabajos de investigaciones anteriores: Es necesario también que los estudiantes desarrollen habilidades para la búsqueda de investigaciones de estudios relacionados. Esta búsqueda de información puede ser en bibliotecas de universidades, bibliotecas virtuales, organismos especializados en estudios económicos, organismos locales, etc. Habitualmente los estudiantes hacen uso de la biblioteca Urania Zelaya de la facultad, haciendo referencia solo de estudios de tesis que realizan los estudiantes en el área económica, lo que limita un poco la comprensión de la temática de estudio.

- La amplitud del tema: Esto hace referencia a la capacidad para seleccionar el tamaño correcto en términos de alcance del estudio, es decir un estudio que sea manejable en el tiempo, en el espacio y con los recursos disponibles. (Blaxter, Cristina, \& Malcom, 2008). Es muy común que los estudiantes deseen el elegir temas de investigación muy amplios sin tomar en cuenta el tiempo que requerirá al recopilar la información y los recursos que necesitarán.

- Tiempo del que dispone: Para llevar a cabo la investigación deben de analizar el tiempo disponible para realizarla, además de tener la capacidad de hacer un uso eficiente del tiempo. El trabajo de investigación como forma de culminación de estudios en la carrera de Economía comprende las siguientes fases: La realización del protocolo de investigación, el cual se realiza en el IX Semestre en la asignatura de investigación aplicada; la recogida y procesamiento de datos, análisis de resultados y la elaboración del informe final; lo que se realiza en X Semestre en la asignatura de seminario de graduación. Los estudiantes de Economía, deben poder hacer un uso eficiente del tiempo, ya que este proceso lo deben realizar paralelo a las otras asignaturas del pensum académico, el cual contempla un semestre de realización de prácticas profesionales en las empresas.

- El costo de la investigación: Para poder realizar el trabajo de investigación los estudiantes deben ser capaces de poder realizar un presupuesto de gastos que incurrirá en la realización del trabajo, ya que este es financiado totalmente por el estudiante.

- Centrarse: Es necesario poder centrar el tema de investigación; lo cual requiere indagación bibliográfica, pensar en el método que se emplearán. Además, deben asegurarse que el tema de estudio tiene una relevancia social. 
- Métodos de investigación: Cuando se elige el tema de investigación es necesario tener en cuenta el método que se utilizará para recabar y analizar los datos. Por ejemplo, si tiene habilidad para comunicarse con la gente se le puede aconsejar que haga uso de la entrevista, si por el contrario se le dificulta comunicarse con la gente es aconsejable las investigaciones documentales, y si lo que quiere es hacer análisis estadísticos o multivariables hay que considerar una metodología cuantitativa. (Blaxter, Cristina, \& Malcom, 2008)

En la carrera de Economía normalmente se plantean estudios cuantitativos, donde se considera los análisis estadísticos como una forma de entender las dinámicas socioeconómicas, haciendo uso de los instrumentos encuesta. Para esto los estudiantes necesitan tener conocimientos sobre estadística tanto como para el cálculo de la muestra como para el procesamiento de la información para lo cual requieren manejar programas estadísticos, tales como SPSS; así mismo el dominio de la hoja de cálculo Excel para procesamiento de los gráficos.

- Investigación grupal o individual: Según Tooter (citado en Blaxter, Cristina \& Malcom, 2008) si estas colgando de una cuerda de doscientos metros, es importante saber que quien se encuentra en el otro extremo es un amigo. La decisión de trabajar en grupo o individual es muy importante porque esto afecta directamente el buen desarrollo del trabajo de investigación, para esto es importante que cada persona conozca bien las cosas que domina, las cosas que no sabe hacer, sus preferencia, motivaciones; dentro del proceso de investigación se pueden encontrar diferencias entre los miembros del grupo para esto los integrantes deben ser capaces de poder gestionar el grupo de investigación; lo que conlleva la división de las responsabilidades, una evaluación del progreso y liderazgo.

La normativa de seminario de graduación indica que los integrantes del grupo deben ser no mayores que tres, por lo que al inicio del curso de investigación aplicada se les orienta la organización de los grupos, recalcando la importancia de formar grupos con personas afines con las cuales puedan organizarse y dividirse las tareas del proceso de investigación; sin embargo, se ha tenido la experiencia de grupos de estudiantes que han decidió separarse a mediados del proceso e incluso casi al finalizar el trabajo de investigación, ya que no poseen la capacidad de poder gestionar el grupo de investigación. Para el que el grupo de investigación funcione eficazmente, los integrantes deben poseer las siguientes capacidades, habilidades, destrezas y cualidades:

- Responsabilidad

- Respeto

- Trabajo en equipo

- Realizar y aceptar críticas constructivas

- Conocer el área de investigación

- Entrega

- Interés

- Capaz de gestionar el tiempo 


\section{CONCLUSIONES}

Un componente muy importante en la formación de los profesionales de la economía lo constituye la investigación científica como eje transversal del proceso de formación profesional, a través del uso de las herramientas que proporcionan las tecnologías de la información y comunicación. (Universidad Nacional Autònoma de Nicaragua, 2012).

En el campo económico es importante que los estudiantes adquirieran ciertas competencias en el campo investigativo, ya que los estudiantes deben poder analizar los fenómenos socioeconómicos a nivel local, nacional, regional e internacional y sus efectos en la económica de Nicaragua, para poder así formular políticas y estrategias que contribuyan al desarrollo económico, la disminución de la pobreza en el país.

Esta competencia en investigación les servirá a los estudiantes en todos los campos laborales en donde se desenvuelvan, ya que pueden ser aplicados para análisis microeconómico: comportamientos de los mercados, análisis de oferta y demanda, entre otros; esto con el fin de darles herramientas en la toma de decisiones empresariales. Así como en el ámbito macroeconómico; tales como investigaciones socioeconómicas de los hogares nicaragüenses de manera que sirvan para la toma de decisiones en política económica.

\section{BIBLIOGRAFÍA}

Blaxter, L., Cristina, H., \& Malcom, T. (2008). Cómo se investiga. Barcelona: Editorial GRAÒ, de IRIF, S.L.

Càzares Aponte, L., \& Cueves de la Garza, J. F. (2008). Planeación y Evaluación basado en competencias. México: Trillas.

Irias, D. C. (2012). Formación de competencias investigativas en las y los estudiantes de la asignatura de ciencias naturales de tercer curso de ciclo común en el Instituto "Gabriela Núñez”. Tegucigalpa: Universidad Pedagógica Nacional Francisco Morazan.

Tovar, L. A. (2011). Las nueve competencias de un investigador.

Universidad Nacional Autónoma de Nicaragua. (2012). Perfil carrera Economia. Managua: UNAN MANAGUA.

Zabala, A., \& Arnau, L. (2008). Cómo aprender y enseñar competencias. Barcelona: Grao. 